University of Nebraska - Lincoln

DigitalCommons@University of Nebraska - Lincoln

Peter Dowben Publications

Research Papers in Physics and Astronomy

December 1996

\title{
Strain-induced distortion of the bulk bands of gadolinium
}

\author{
Carlo Waldfried \\ University of Nebraska-Lincoln \\ D.N. Mcllroy \\ University of Nebraska-Lincoln \\ C.W. Hutchings \\ University of Nebraska-Lincoln \\ Peter A. Dowben \\ University of Nebraska-Lincoln, pdowben@unl.edu
}

Follow this and additional works at: https://digitalcommons.unl.edu/physicsdowben

Part of the Physics Commons

Waldfried, Carlo; Mcllroy, D.N.; Hutchings, C.W.; and Dowben, Peter A., "Strain-induced distortion of the bulk bands of gadolinium" (1996). Peter Dowben Publications. 43.

https://digitalcommons.unl.edu/physicsdowben/43

This Article is brought to you for free and open access by the Research Papers in Physics and Astronomy at DigitalCommons@University of Nebraska - Lincoln. It has been accepted for inclusion in Peter Dowben Publications by an authorized administrator of DigitalCommons@University of Nebraska - Lincoln. 


\title{
Strain-induced distortion of the bulk bands of gadolinium
}

\author{
Carlo Waldfried, D. N. McIlroy, C. W. Hutchings, and P. A. Dowben \\ Department of Physics \& Astronomy and the Center for Materials Research and Analysis, Behlen Laboratory of Physics, University of \\ Nebraska-Lincoln, Lincoln, Nebraska 68588-0111
}

(Received 28 June 1996)

\begin{abstract}
Thin films of gadolinium, approximately $8 \mathrm{ML}$ thick, have been grown on the corrugated (112) surface of molybdenum and the electronic structure has been investigated with angle-resolved photoelectron spectroscopy. The unfavorable lattice match between the "steplike"' Mo(112) substrate and the preferred hexagonally ordered Gd film results in an incommensurate Gd structure that appears to be ordered, but strained along the direction of the corrugations. The hexagonal Gd lattice is expanded by more than $20 \%$ along the "step" lines of the substrate, as determined from the reduced Brillouin-zone size along the $\overline{\Gamma \Sigma M}$ high-symmetry line and low-energy electron diffraction. The induced strain substantially alters the conventional Gd $5 d / 6 s$ bulk bands to exhibit a dispersion opposite to that of the relaxed $\operatorname{Gd}(0001)$ structure. Dislocations destroy the long-range crystallographic order in the direction orthogonal to the corrugations, which results in the localization of the bands along the $\overline{\Gamma T K}$ symmetry line. [S0163-1829(96)00848-X]
\end{abstract}

The modification of crystalline films of $3 d$ transition metals in terms of lattice expansions, or contractions, can significantly alter the electronic structure. ${ }^{1,2}$ Some calculations exist for the strained electronic structure of the rare earths [in particular, of Pr (Ref. 3)]. Nonetheless, only a few experimental studies have been performed as a result of the considerable difficulties in preparing strained, crystalline films. ${ }^{4,5}$ Small amounts of strain are obtained for ultrathin films of $\mathrm{Gd}(0001)$ grown on $\mathrm{W}(110),{ }^{6}$ but only few differences were observed in the electronic structure which could be attributed to strain. ${ }^{4}$

The experimental band structure of hexagonal closedpacked Gd has been studied extensively. ${ }^{4,5,7-12}$ The bulk band structure resembles Stoner-like behavior, ${ }^{8,13,14}$ while the surface exhibits a temperature-dependent electronic structure similar to a rigid-band system. ${ }^{14}$ The valenceand/or conduction-band electrons are itinerant and are believed to mediate the magnetic exchange coupling of the large local moments of the half-filled $4 f$ core levels $\left(4 f^{7} 5 d^{1} 6 s^{2}\right){ }^{15}$ The magnetic coupling to the nearby atoms is established by partial polarization of the $5 d / 6 \mathrm{~s}$ valence and/or conduction electrons (indirect coupling). Strain is certainly expected to alter the band structure of the valence and conduction electrons, which will consequently have pronounced effects on the magnetic properties. The effect of strain on the electronic structure of $\mathrm{Gd}$ is therefore of fundamental interest. In this paper we will discuss the modified valence-band electronic structure of strained thin $\mathrm{Gd}$ films (8-10 ML), which were obtained by growing Gd on a corrugated $\mathrm{Mo}(112)$ substrate (with the atomic distance perpendicular to the Mo furrow direction of $4.45 \AA$ ). The experimental band structure was mapped using angle resolved ultraviolet photoemission spectroscopy and the structural analysis was undertaken with low-energy electron diffraction (LEED).

The photoemission experiments were carried out in a UHV chamber equipped with a hemispherical electron energy analyzer with an angular acceptance of $\pm 1^{\circ} .{ }^{16}$ The light was dispersed with a 6-m toroidal grating monochromator at the Synchrotron Radiation Center in Stoughton, Wisconsin. The combined energy resolution was $\sim 150 \mathrm{meV}$. The sample temperature was monitored with a Re/W 5-26\% thermocouple with an accuracy of $\pm 5 \mathrm{~K}$. All photoemission spectra shown in this paper have been acquired at $T \approx 150 \mathrm{~K}$ with a photon energy of $35 \mathrm{eV}$ at an incident angle of $45^{\circ}$.

Thin Gd films of 20-25 $\AA$ were grown at approximately $150 \mathrm{~K}$ on a $\mathrm{Mo}(112)$ crystal by slow thermal deposition. The chamber pressure during deposition was less than $1.5 \times 10^{-10}$ Torr, while the base pressure was in the $9 \times 10^{-11}$ Torr range. The Gd film thickness was monitored with a quartz-crystal oscillator that was calibrated by monitoring the attenuation of the molybdenum $4 p$ core-level signal and the increased signal of the $\operatorname{Gd} 4 f$ core levels. The as grown films were subsequently annealed at approximately $500 \mathrm{~K}$ for $5 \mathrm{~min}$ to maximize structural order, as indicated by the development of the bulk band dispersion and LEED.

Low-temperature annealing $(T<500 \mathrm{~K})$ of the asdeposited Gd films gives an imperfect $(1 \times 1)$ LEED pattern [Fig. 1(b)]. The well-ordered Gd films grown on Mo(112) exhibit a streaked hexagonal LEED pattern, where the streaks are oriented perpendicular to the substrate "step", lines [Fig. 1(a)]. This indicates that the Gd films order in a hexagonal structure along the corrugation lines. The streaks imply that misfit dislocations form perpendicular to the "step" direction and propagate to the surface of the 8-10ML-thick Gd films. The ordering of the Gd films is associated with a narrowing of the surface state linewidth, as well as the formation of the $\mathrm{Gd} 5 d / 6 \mathrm{~s}$ bulk bands.

Both the strain and the misfit dislocations within the thin Gd films with hexagonal order are manifest in the band dispersion of the $5 d / 6 \mathrm{~s}$ bulk bands. Figure 2 shows two sets of valence-band photoemission spectra that were acquired for various points along the high-symmetry lines $\overline{\Gamma \Sigma M}$ (top) and $\overline{\Gamma T K}$ (bottom) of the surface Brillouin zone of the strained Gd film. The normal-emission spectra are composed of the Gd $4 f$ core level at a binding energy of $8.4 \mathrm{eV}$, the $\mathrm{Gd}$ $5 d / 6 s$ bulk bands at $\sim 1.8 \mathrm{eV}$ below $E_{F}$ and the $\mathrm{Gd}$ 

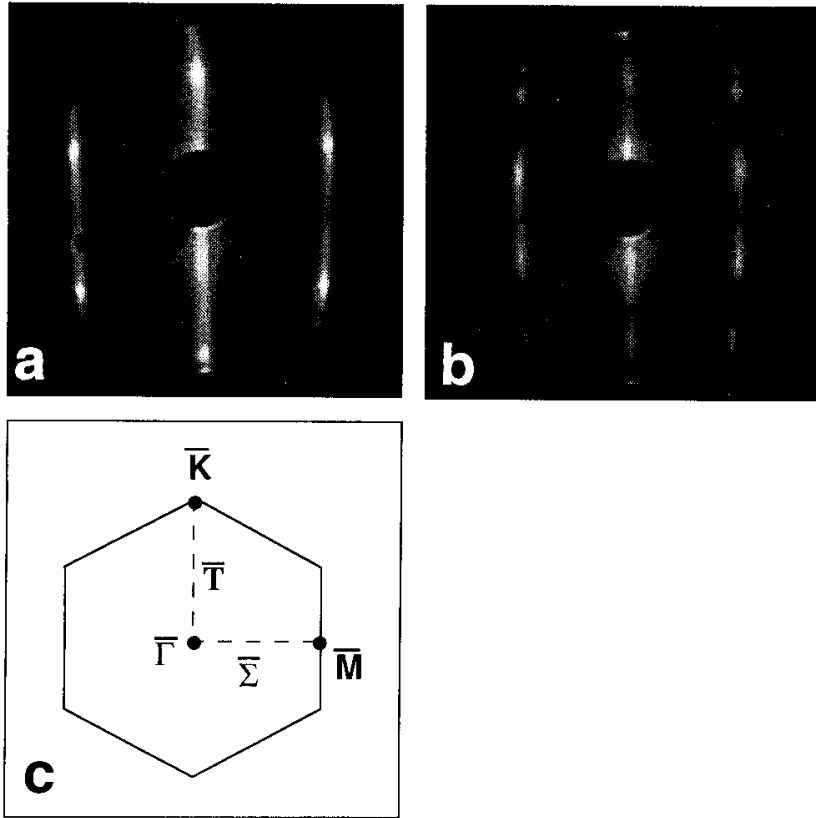

FIG. 1. The LEED pictures of a 25 - $\AA$-thick Gd film grown on $\mathrm{Mo}(112)$ and annealed at $650 \mathrm{~K}$ (a) and $480 \mathrm{~K}$ (b), which were acquired with electron energies of 44.6 and $58.0 \mathrm{eV}$, respectively. The LEED pattern (a) implies a hexagonal ordered structure with the surface Brillouin zone displayed in panel (c). The streaks are in the direction perpendicular to the substrate corrugations.

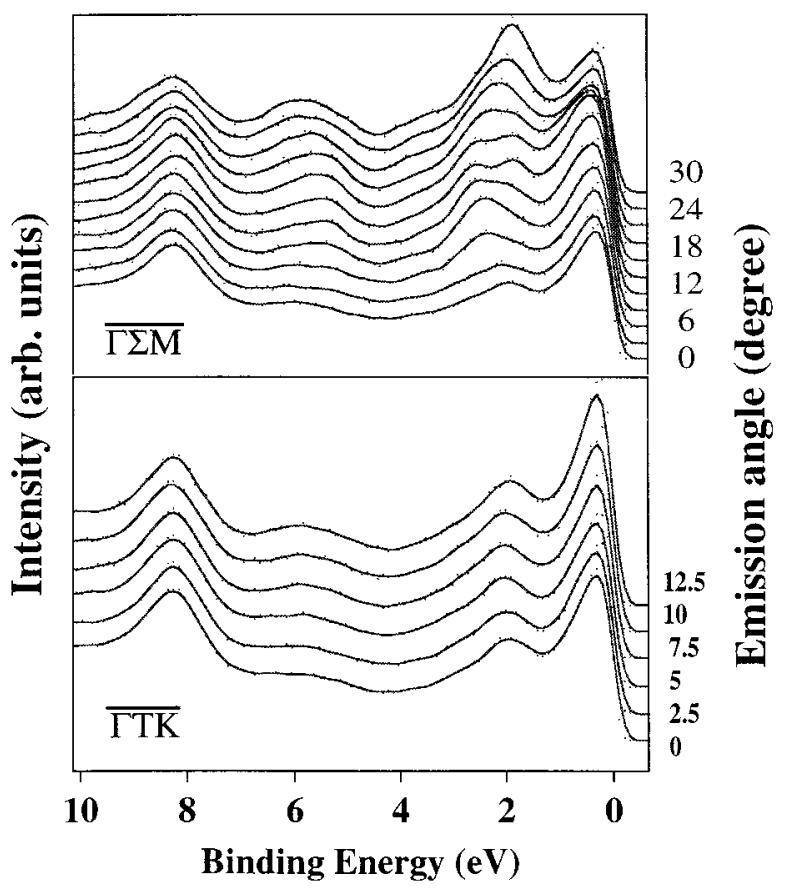

FIG. 2. The emission angle-dependent photoemission spectra of a $25-\AA \mathrm{Gd}$ film grown on $\mathrm{Mo}(112)$ along the $\overline{\Gamma \Sigma M}$ (top) and $\overline{\Gamma T K}$ (bottom) high-symmetry directions of the surface Brillouin zone. The spectra were acquired at $150 \mathrm{~K}$ with a photon energy of $35 \mathrm{eV}$.

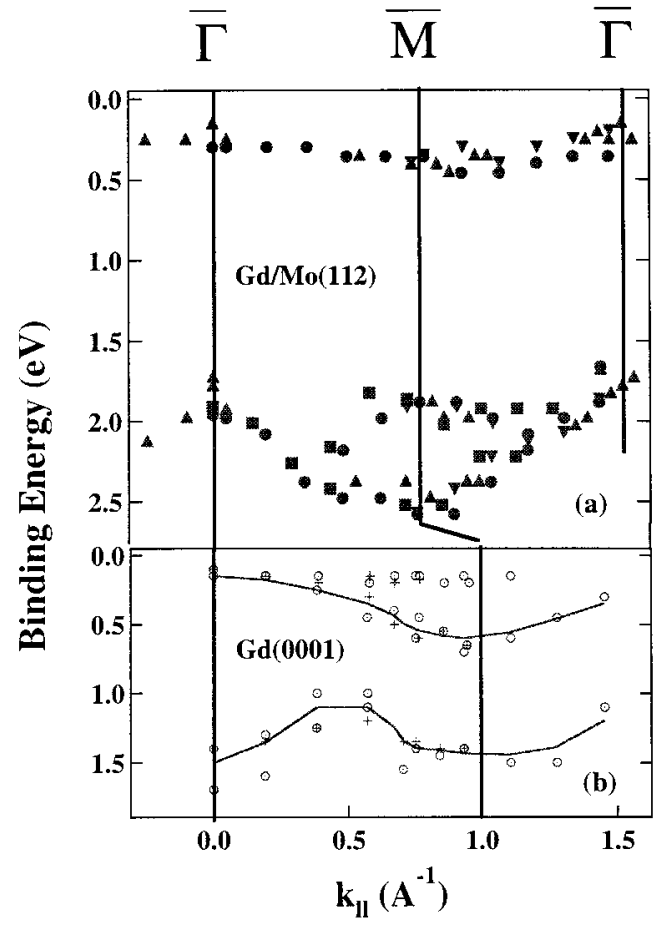

FIG. 3. (a) The band dispersion of strained Gd film along the $\overline{\Gamma \Sigma M}$ direction of the Brillouin zone. The figure was constructed from data acquired from films of $20-\AA$ nominal thickness which were annealed to $495 \mathrm{~K}(\boldsymbol{\square}), 508 \mathrm{~K}(\boldsymbol{O})$ and $521 \mathrm{~K}(\boldsymbol{\Delta})$, as well as a 25 - $\AA$ film annealed at $508 \mathrm{~K}(\boldsymbol{\nabla})$. (b) The experimental band dispersion of an ordered $\mathrm{Gd}(0001)$ film grown on W(110) along $\overline{\Gamma \Sigma M}$ of the surface Brillouin zone. The data was reduced from Refs. 4 and 11.

$5 d_{z^{2}-r^{2}}$ surface state at $\sim 0.2-\mathrm{eV}$ binding energy. At normal emission the valence-band spectrum of the strained $\mathrm{Gd}$ film closely resembles the photoemission spectrum of the unstrained $\operatorname{Gd}(0001),{ }^{4,7-12}$ with the exception of the markedly broader surface state $^{9,11} \quad\left(\mathrm{FWHM}_{\mathrm{Gd} / \mathrm{Mo}(112)} \approx 1.3 \mathrm{eV}\right.$, $\left.\mathrm{FWHM}_{\mathrm{Gd}(0001)} \approx 0.2 \mathrm{eV}\right)$. A well-defined very intense surface state is characteristic of the clean hexagonal ordered Gd(0001) surface. ${ }^{4,9,11}$ The broadening and suppression of the surface state of the strained Gd films may be indicative of several things: hybridization of the surface state with the bulk bands, ${ }^{17}$ imperfect surface order, or an alteration of the symmetry of the surface, relative to the unstrained $\mathrm{Gd}(0001)$.

Both the $5 d / 6 s$ bulk bands and the surface state of the dislocated and strained Gd films exhibit pronounced band dispersion (bandwidth: $\Delta E_{\text {bulk }} \approx 0.8 \mathrm{eV}, \Delta E_{\text {surface }} \approx 0.25 \mathrm{eV}$ ) along the $\overline{\Gamma \Sigma M}$ direction (parallel to the substrate corrugations) of the SBZ [Fig. 2(a)]. In contrast, the Gd $5 d / 6 \mathrm{~s}$ valence bands are completely dispersionless along the $\overline{\Gamma T K}$ high-symmetry line (orthogonal to the substrate corrugations). The absence of the dispersion along the $\overline{\Gamma T K}$ direction, coupled with the lack of changes in the intensities with emission angle [Fig. 2(b)] indicates that the film has no longrange order perpendicular to the substrate corrugations. This is consistent with the formation of dislocations perpendicular to the corrugations, as indicated by the streaks in the LEED pattern.

The effects of strain within the thin Gd films is clearly 
reflected in the reduced Brillouin-zone size of the $\mathrm{Gd} /$ $\mathrm{Mo}(112)$ films as compared to unstrained $\operatorname{Gd}(0001)$. The band dispersion along the $\overline{\Gamma \Sigma M}$ high-symmetry line of the strained Gd films are displayed Fig. 3(a). For comparison the band dispersion of unstrained $\mathrm{Gd}(0001)$ is presented in Fig. 3 (b). Based on the dispersion of the strained Gd films in Fig. 3(a), we have determined the zone edge $\bar{M}$ to be at $0.77 \AA^{-1}$, which is smaller than that of unstrained $\operatorname{Gd}(0001)$ $\left(\bar{\Gamma}-\bar{M}=1.0 \AA^{-1}\right) .^{4,5,11,12}$ This implies a relaxation of the hexagonal $\mathrm{Gd}$ structure along the substrate corrugation lines of more than $20 \%$. This yields a relationship between the lattice vectors of $\mathrm{Gd}$ and Mo of $2: 3 \quad\left(a_{\mathrm{Mo}}=2.73 \AA\right.$ and $\left.a_{\mathrm{Gd}}=4.09 \AA\right)$. A lattice vector relationship of $n: 1$ has been observed for overlayers of $\mathrm{Mg},{ }^{18} \mathrm{Cs},{ }^{19}$ and $\mathrm{Na}$ (Ref. 20) on the $\mathrm{Mo}(112)$ surface; therefore the above relationship is not without precedence.

The differences between the band structure of the strained Gd film and the unstrained $\mathrm{Gd}(0001)$ along the $\overline{\Gamma \Sigma M}$ direction of the SBZ are dramatic. The Gd surface state of the strained film disperses away from the Fermi level in a fashion similar to that of the surface state of well-ordered unstrained Gd films. The $5 d / 6 s$ bulk bands of the strained Gd films, however, disperse downward (to higher binding energy with increasing wave vector), which is in the opposite direction of their counterparts in unstrained epitaxial $\mathrm{Gd}$ films ${ }^{7-12}$ which disperse upwards towards the Fermi level along $\overline{\Gamma \Sigma M}$ direction [Fig. 3(b)]. In addition, there are at least two bulk bands for the $\mathrm{Gd} / \mathrm{Mo}(112)$ films. At least one band is located at approximately $1.8 \mathrm{eV}$ below $E_{F}$ at $\bar{\Gamma}$ and disperses downwards to higher binding energies away from the zone center. Two bulk bands are observed beginning approximately half way across the Brillouin zone. At the $\bar{M}$ point these two bands are separated by $\sim 0.8 \mathrm{eV}$, where the lower band is at a binding energy of $2.6 \mathrm{eV}$.

Although the symmetry of the two bulk bands of the strained Gd films are even with respect to the mirror plane, as is the case for unstrained $\mathrm{Gd}(0001)$, the orbital symmetry of these two strained Gd $5 d, 6 s$ bulk bands may still be different from that of the unstrained $\operatorname{Gd}(0001)$. There are several possible origins for the observed band structure of the strained Gd films: (i) The two bands may be composed of spin minority and spin majority bands, which are of $5 d_{z^{2}-r^{2}}$ character, similar to the $\mathrm{Gd}(0001)$. Unlike the Gd(0001), however, two strained Gd bands may degenerate at the Brillouin-zone center and exhibit exchange splitting only near the zone edge, possibly due to altered magnetic ordering or reduced symmetry. (ii) The increased atomic spacing in the strained Gd may favor the wave function overlap of $\mathrm{Gd} 5 d_{x z, y z}$ or $5 d_{x^{2}-y^{2}}$ orbitals, rather than the Gd $5 d_{z^{2}-r^{2}}$. This would suggest an assignment of the two bulk bands in the strained Gd valence band as the $5 d_{x z}$ and $5 d_{y z}$, and/or $5 d_{x^{2}-y^{2}}$ bands, which may form a bondingantibonding pair. One band $\left(5 d_{x^{2}-y^{2}}\right)$ may not be easily observed at $\Gamma$ because of symmetry selection rules. (iii) The expanded Gd lattice may result in a more atomiclike valenceband structure, with a splitting of the bands into mainly $6 \mathrm{~s}$ and $5 d$ character near the zone edge. Our suggestions, however, leave the questions about the origin of the modified band structure of the strained Gd films unanswered. Theoretical band structures, which are lacking at this point, are needed for strained layers of Gd. We expect that the strain modified band structure may reflect changes in the magnetic properties. If theory is to be believed, these strained and expanded lattice films will remain ferromagnetically ordered as predicted by calculations by Eriksson et al. ${ }^{22}$ and Harmon et al. ${ }^{21}$ rather than changing to antiferromagnetic ordering.

In conclusion we have investigated the electronic and structural properties of strained Gd films, as grown on the corrugated Mo(112) substrate. The Gd films are ordered and strained along the substrate corrugation lines, but have high densities of dislocations perpendicular to the corrugation direction, which in turn breaks down the long-range order in this direction. The strain is reflected by a hexagonal $\mathrm{Gd}$ structure that is expanded by at least $20 \%$ in the direction along the "steps." As a consequence of strain, the Gd $5 d / 6 s$ bulk bands disperse in the opposite direction of those of the unstrained $\operatorname{Gd}(0001)$. At least one band is located $1.8 \mathrm{eV}$ below $E_{F}$ at $\bar{\Gamma}$ and uniaxially disperses along $\overline{\Gamma \Sigma M}$ towards higher binding energy. Two bulk bands are observed in the vicinity of the $\bar{M}$ with a maximum energy splitting of $\sim 0.8$ $\mathrm{eV}$. The strained Gd films exhibit anisotropies in the band structure and in the long-range order. We do not, as yet, know how strain will affect the magnetic ordering, or how the strain and misfit dislocations will influence the Curie or Neèl temperature of the Gd films, but these are issues of great interest.

This work was supported by NSF through grant Nos. DMR-92-21655 and DMR-94-96131. The experiments were carried out at the Synchrotron Radiation Center which is also funded by NSF.
${ }^{1}$ V. L. Moruzzi, P. M. Marcus, K. Schwarz, and P. Mohn, Phys. Rev. B 34, 1784 (1986).

${ }^{2}$ P. A. Dowben, S. Varma, Y. J. Kime, D. R. Mueller, and M. Onellion, Z. Phys. B 73, 247 (1988).

${ }^{3}$ S. K. De and S. Chatterjee, J. Phys. F 17, 2057 (1987); M. Dakshinamoorthy, K. Iyakutti, S. Sankar, and R. Asokamani, Z. Phys. B Condens. Matter 55, 299 (1984); S. De, I. Bose, and S. Chatterjes, Phys. Status Solidi B 127, 605 (1985); D. Glötzel, Physics of Solids Under High Pressure, edited by J. S. Schilling and R. N. Shelton (North Holland, Amsterdam, 1981), p. 263; A. K. Mcmahan, H. L. Shriver, and B. Johansson, Physics of Solids Under High Pressure, edited by J. S. Schilling and R. N.
Shelton (North Holland, Amsterdam, 1981), p. 169.

${ }^{4}$ P. A. Dowben, D. N. McIlroy, and Dongqi Li, in Handbook on the Physics and Chemistry of He Rare Earths, edited by K. A. Gschneider and LeRoy Eyring (North Holland Press, Amsterdam, 1997), Vol. 24, Chap. 159.

${ }^{5}$ S. D. Barrett, Surf. Sci. Rep. 14, 271 (1992).

${ }^{6}$ D. Weller and S. F. Alvarado, J. Appl. Phys. 59, 2908 (1986).

${ }^{7}$ F. J. Himpsel and B. Reihl, Phys. Rev. B 28, 574 (1983).

${ }^{8}$ Bongsoo Kim, A. B. Andrews, J. L. Erskine, Kwang Joo Kim, and B. N. Harmon, Phys. Rev. Lett. 68, 1931 (1992).

${ }^{9}$ Dongqi Li, C. W. Hutchings, P. A. Dowben, C. Hwang, RongTzong Wu, M. Onellion, A. B. Andrews, and J. L. Erskine, J. 
Magn. Magn. Mater. 99, 85 (1991).

${ }^{10}$ Dongqi Li, Jiandi Zhang, P. A. Dowben, Rong-Tzong Wu, and M. Onellion, J. Phys. Condens. Matter 4, 3929 (1992).

${ }^{11}$ Dongqi Li, Jiandi Zhang, P. A. Dowben, and M. Onellion, Phys. Rev. B 45, 7272 (1992).

${ }^{12}$ Dongqi Li, P. A. Dowben, J. E. Ortega, and F. J. Himpsel, Phys. Rev. B 49, 7734 (1994).

${ }^{13}$ W. Nolting, T. Dambeck, and G. Borstel, Z. Phys. B 94, 409 (1994).

${ }^{14}$ Dongqi Li, J. Pearson, S. D. Bader, D. N. McIlroy, C. Waldfried, and P. A. Dowben, Phys. Rev. B 51, 13895 (1995).

${ }^{15}$ Dongqi Li, Jiandi Zhang, P. A. Dowben, and M. C. Onellion, Phys. Rev. B 48, 5612 (1993).

${ }^{16}$ P. A. Dowben, D. LaGraffe, and M. Onellion, J. Phys. Condens. Matter 1, 6571 (1989).

${ }^{17}$ Dongqi Li, C. W. Hutchings, P. A. Dowben, Ron-Tzong Wu, C.
Hwang, M. Onellion, A. B. Andrews, and J. L. Erskine, J. Appl. Phys. 70, 6565 (1991).

${ }^{18}$ Jiandi Zhang, D. N. McIlroy, and P. A. Dowben, Phys. Rev. B 49, 13780 (1994).

${ }^{19}$ M. S. Gupalo, V. K. Medvedev, B. M. Palyukh, T. P. Smereka, Fiz. Tverd. Tela (Leningrad) 23, 2076 (1981) [Sov. Phys. Solid State 23, 1211 (1981)].

${ }^{20}$ M. S. Gupalo, V. K. Medvedev, B. M. Palyukh, and T. P. Smereka, Fiz. Tverd. Tela (Leningrad) 22, 3201 (1980) [Sov. Phys. Solid State 22, 1873 (1981)].

${ }^{21}$ B. N. Harmon, V. P. Antropov, A. I. Liechtenstein, J. V. Solovyev, and V. I. Anisimov, J. Phys. Chem. Solids 56, 1521 (1995).

${ }^{22}$ O. Eriksson, R. Ahuja, A. Ormeci, J. Trygg, O. Hjorststam, P. Söderlind, B. Johansson, and J. M. Wills, Phys. Rev. B 52, 4420 (1995). 